\title{
Properties of Non-Tilted Bent-Core Orthogonal Smectic Liquid Crystal
}

S. Sreenilayam

Trinity College Dublin, Ireland

M. Nagaraj

Technological University Dublin

Yuri Panarin

Technological University Dublin, yuri.panarin@tudublin.ie

See next page for additional authors

Follow this and additional works at: https://arrow.tudublin.ie/engscheleart2

Part of the Electrical and Computer Engineering Commons

\section{Recommended Citation}

Lehmann, A. et al. (2012). Properties of non-tilted bent-core orthogonal smectic liquid crystal. Molecular Crystals and Liquid Crystals, vol. 553, pg. 140-146. DOI:10.1080/15421406.2011.609457

This Article is brought to you for free and open access by the School of Electrical and Electronic Engineering at ARROW@TU Dublin. It has been accepted for inclusion in Articles by an authorized administrator of ARROW@TU Dublin. For more information, please contact arrow.admin@tudublin.ie, aisling.coyne@tudublin.ie, gerard.connolly@tudublin.ie.

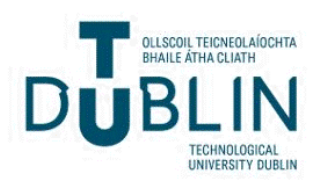




\section{Authors}

S. Sreenilayam, M. Nagaraj, Yuri Panarin, J. K. Vij, A. Lehmann, and C. Tschierske

This article is available at ARROW@TU Dublin: https://arrow.tudublin.ie/engscheleart2/159 


\title{
Properties of Non-Tilted Bent-Core Orthogonal Smectic Liquid Crystal
}

\author{
S. SREENILAYAM, ${ }^{1}$ M. NAGARAJ ${ }^{2}$ Y. P. PANARIN,${ }^{1,2, *}$
} J. K. VIJ, ${ }^{2}$ A. LEHMANN, ${ }^{3}$ AND C. TSCHIERSKE ${ }^{3}$

${ }^{1}$ Department of Electronic and Electrical Engineering, Trinity College Dublin, Dublin 2, Ireland

${ }^{2}$ School of Electronic \& Communication Engineering, Dublin Institute of Technology, Dublin, Ireland

${ }^{3}$ Institute of Organic Chemistry, Martin Luther-University Halle-Wittenberg, D 06120, Germany

\begin{abstract}
We present the properties of different achiral orthogonal polar smectic phases observed in a homologous series of bent-core molecular compounds. The macroscopically uniaxial SmAP $P_{R}$ phase transforms to biaxial state by the application of higher electric fields and biaxial $S m A P_{A}$ phase undergoes biaxial-uniaxial-biaxial texture transformation with increasing electric fields, i.e., initial antiferroelectric structure transforms into ferroelectric state under higher electric fields and it shows three different optically distinguishable states. The $S m A P_{A R}$ phase shows an entirely different response compared to other polar smectic phases under study. The dielectric measurements in the $S m A P_{A R}$ phase is the evidence of antiferroelectric behavior of uniaxial state. The polarizing microscopy texture observation supports the corresponding biaxiality measurements.
\end{abstract}

Keywords Bent-core molecules; orthogonal biaxial polar Smectic; electro-optic effects; dielectric relaxation; polarization

\section{Introduction}

After the discovery of ferroelectricity in tilted smectic phases of chiral molecules by Robert Mayer et al. in 1975, the idea of having a spontaneous electric polarization in the liquid crystalline state was revolutionary [1] and a model of ferroelectricity for an orthogonal smectic phase $(\mathrm{Cp})$ formed by non-chiral bent core molecules were recommended by Brad et al. in 1995 [2]. In 1996, Niori et al. reported the experimental proof of ferroelectricity for the first time [3].

In addition to conventional banana phases such as B1-B8 [4] orthogonal smectic phases and biaxial nematic $\left(\mathrm{N}_{\mathrm{b}}\right)$ phases have been reported. The bent core materials consist of biaxial nematic phase offers fast switching by the rotation of secondary short axis $m$ around the fixed long axis $n$ due to the coupling of dielectric anisotropy $\left(\varepsilon_{\mathrm{m}}-\varepsilon_{1}\right)$ with the fields. Although the $\mathrm{N}_{\mathrm{b}}$ phase has offered some newer possibilities in display applications, it is very difficult to obtain an independent short axis $(m)$ switching and this is usually accompanied by unnecessary tilt of the primary director.

*Address correspondence to Y. P. Panarin, Department of Electronic and Electrical Engineering, Trinity College Dublin, Dublin 2, Ireland. Email: yuri.panarin@dit.ie 
A homologous series of achiral orthogonal Sm-A like phases have been synthesized and studied recently [4-8]. Upon the reduction of temperature, some bent core molecules exhibit a number of lower temperature polar orthogonal $\operatorname{SmAP}_{R}, \operatorname{SmAP}_{A R}$ and $\operatorname{SmAP}_{A}$ phases in addition to conventional non-polar uniaxial $\mathrm{Sm}$-A phase. Under crossed polarizers in $\mathrm{SmAP}_{\mathrm{R}}$ phase, dipoles form small polar domains and the direction of polar director is randomly arranged in layers in the absence of electric field, leading to macroscopic uniaxial texture and exhibit polar switching. Recently, Gomola et al. reported $\operatorname{SmAP}_{\mathrm{AR}}$ phase in asymmetrical bent-core compound containing 3-aminophenol central unit [5]. This paper presents the physical properties of different non-tilted orthogonal polar smectic phases observed in a homolog series of 4-cyanoresorcinol bisbenzoate with two terephthalatebased wing compounds.

\section{Experimental}

The measurements are carried out in three different 4-cyanoresorcinol bisbenzoate compounds with two terephthalate-based wings of different chain lengths, synthesized in Halle, Germany. The molecular structure of LC materials is shown in Fig. 1 and Table 1 lists the orthogonal achiral smectic compounds investigated. Among these LC materials PAL30 compound exhibits of all three polar orthogonal $\operatorname{SmAP}_{\mathrm{R}}, \operatorname{SmAP}_{\mathrm{AR}}$ and $\mathrm{SmAP}_{\mathrm{A}}$ phases.

The homeotropic alignment is obtained by two glass substrates coated with polymer AL60702 (JSR Korea), for electro optic measurements. The electrodes are arranged to apply in-plane electric field of frequency $110 \mathrm{~Hz}$ by etching indium tin oxide (ITO) stripes on the bottom substrate and the electrode distance is $\sim 80 \mu \mathrm{m}$. A tilting optical compensator is used for biaxiality measurement so that it can take extremely small optical retardation. As the smectic layers lie parallel to the substrate the homeotropic cell shows perfect alignment of very large extinction on cooling from Isotropic to $\mathrm{SmA}$ and then to $\mathrm{SmAP}_{\mathrm{R}}$ phases. The dielectric study is carried out using broadband high resolution dielectric spectrometer (Novocontrol GmbH, Germany) on planar cells of $10 \mu \mathrm{m}$ thickness.

\section{Results and Discussion}

\subsection{Textures and Biaxiality in a Homeotropic Cells}

The effect of electric field on different orthogonal smectic phases has been studied in a 7 $\mu \mathrm{m}$ homeotropic cell under crossed polarizers with an angle of $45^{\circ}$ between the electric field and polarizer axis.

Figure 2 presents the electric field of induced biaxiality $\operatorname{SmAP}_{\mathrm{A}}$ phase in PAL31 material.

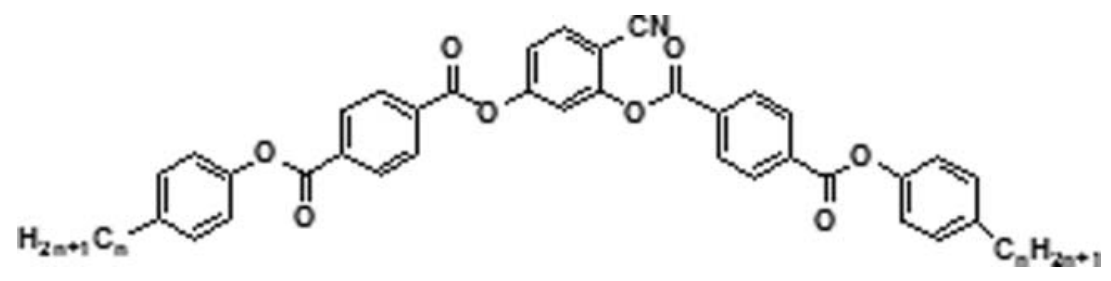

Figure 1. Chemical structure of orthogonal achiral smectic liquid crystals. 
Table 1. List of orthogonal achiral smectic materials under investigation.

\begin{tabular}{lcc}
\hline Material & Chain length & \multicolumn{1}{c}{ Phase sequence } \\
\hline PAL 31 & 8 & SmAP $_{\mathrm{A}} 121^{\circ} \mathrm{C} \mathrm{SmA} 163^{\circ} \mathrm{C} \mathrm{I}$ \\
CK 64 & 12 & SmAP $_{\mathrm{A}} 111^{\circ} \mathrm{C} \mathrm{SmAPz} 157.5^{\circ} \mathrm{C} \mathrm{SmA} 163.9^{\circ} \mathrm{C} \mathrm{I}$ \\
PAL 30 & 14 & $\mathrm{SmAP}_{\mathrm{A}} 95^{\circ} \mathrm{C} \mathrm{SmAPz} 108^{\circ} \mathrm{C} \mathrm{SmAP}_{\mathrm{AR}} 111^{\circ} \mathrm{C}$ \\
& & $\mathrm{SmA} 164^{\circ} \mathrm{C} \mathrm{I}$ \\
\hline
\end{tabular}

The properties of $\mathrm{SmAP}_{\mathrm{A}}$ and $\mathrm{SmAP}_{\mathrm{R}}$ phases were studied in CK 64 material $(\mathrm{n}=12)$. Figure 3 presents the field induced biaxiality measurements and textures in homeotropically aligned cell.

When CK 64 sample is cooled down from SmA to, $\operatorname{SmAP}_{\mathrm{R}}$ phase the sample looks dark under crossed polarized state. Since the polar (secondary) directors in different smectic layers are randomly ordered in the absence of electric field and the primary director is orthogonal to the smectic layer plane, this $\operatorname{SmAP}_{\mathrm{R}}$ phase $[8,10]$ is macroscopically uniaxial. An application of electric field disturbs the random distribution of the secondary director and therefore induces optical biaxiality. The value of induced biaxiality increases with lowering temperature and is stronger near the phase transition to $\mathrm{SmAP}_{\mathrm{A}}$ (compare $116^{\circ} \mathrm{C}$ and $112^{\circ} \mathrm{C}$ ).

While cooling down from $\operatorname{SmAP}_{\mathrm{R}}$ phase to $\mathrm{SmAP}_{\mathrm{A}}$ the Schlieren texture with $\mathrm{s}=1$, $1 / 2$ declinations appears at $110^{\circ} \mathrm{C}$. In this phase, the application of in-plane electric field of $100 \mathrm{~Hz}$ shows a transformation of Schlieren in to biaxial bright state corresponding to antiferroelectric structure with $\delta \mathrm{n}_{\mathrm{AF}}=0.0035$. The further application of field shows a transformation to the dark uniaxial intermediated level. In this uniaxial state the secondary directors in the neighboring smectic layers perpendicular to one another are resulting in macroscopically uniaxial structure [9]. On further increase in field, this uniaxial dark state transforms in to bright biaxial ferroelectric state with $\delta \mathrm{n}_{\mathrm{F}}=0.012$. In higher temperature range of $\operatorname{SmAP}_{\mathrm{A}}$ phase $\delta \mathrm{n}_{\mathrm{AF}}<\delta \mathrm{n}_{\mathrm{AF}}$. With reduction in temperature the biaxiality $\delta \mathrm{n}_{\mathrm{AF}}$

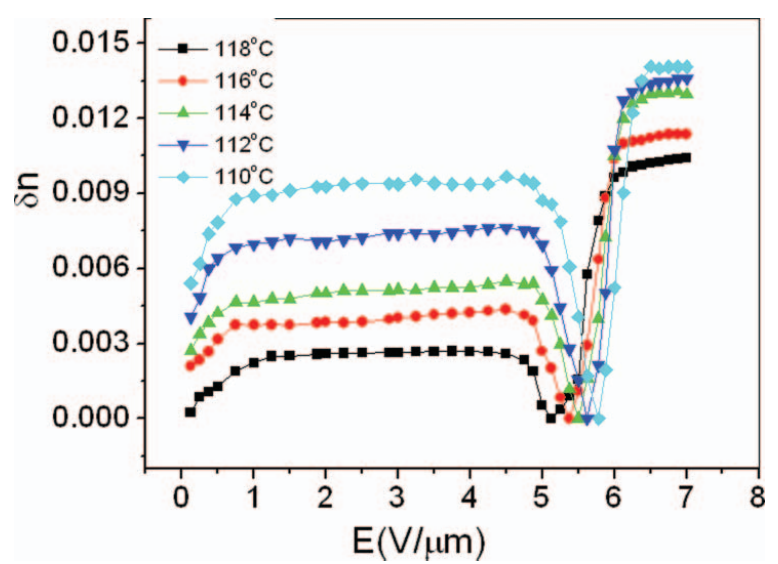

Figure 2. Electric field induced biaxiality measurements in $\mathrm{SmAP}_{\mathrm{A}}$ phase of PAL 31 material with decreasing of temperature (cell thickness $7.6 \mu \mathrm{m}$ and $\mathrm{f}=100 \mathrm{~Hz}$ ). 


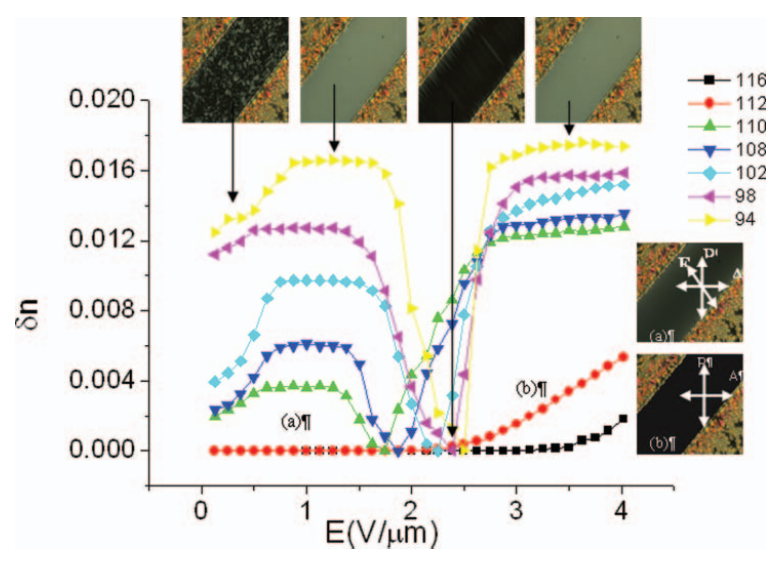

Figure 3. Electric field induced biaxiality measurements in CK 64 material with decreasing of temperature and the $\mathrm{POM}$ textures corresponding to the biaxiality values at $112^{\circ} \mathrm{C}$ and $94^{\circ} \mathrm{C}$ (cell thickness $7 \mu \mathrm{m}$ and $\mathrm{f}=100 \mathrm{~Hz}$ ).

increases faster than $\delta \mathrm{n}_{\mathrm{F}}$ and at $94^{\circ} \mathrm{C}$ the biaxiality values are almost equal for both antiferroelectric and ferroelectric states, $\delta \mathrm{n}_{\mathrm{AF}}=\delta \mathrm{n}_{\mathrm{AF}}$.

The material PAL $30(n=14)$, shows another new phase $\mathrm{SmAP}_{\mathrm{AR}}$ in the temperature range $\left(96^{\circ} \mathrm{C}-109^{\circ} \mathrm{C}\right)$ in addition to $\mathrm{SmAP}_{\mathrm{R}}$ and $\mathrm{SmAP}_{\mathrm{A}}$ phases. The optical texture observations in $\mathrm{SmAP}_{\mathrm{A}}$ and $\mathrm{SmAP}_{\mathrm{A}}$ phases are similar to the corresponding field induced biaxiality measurement for CK-64 (Fig 3). The electric field effect of new phase SmAP $\mathrm{AR}_{\mathrm{AR}}$ with reduction in temperature is shown in Fig. 4.

When the homeotropically aligned cell is cooled down from $\operatorname{SmAP}_{\mathrm{R}}$ to $\mathrm{SmAP}_{\mathrm{AR}}$ phase at $104^{\circ} \mathrm{C}$, it remains completely dark under crossed polarizers for the applied electric field less than $\sim 0.95 \mathrm{~V} / \mu \mathrm{m}$. On the application of in-plane electric field $\sim 1 \mathrm{~V} / \mu \mathrm{m}$ it transforms to the biaxial (bright) state with $\delta \mathrm{n}=0.002$ up to $1.2 \mathrm{~V} / \mu \mathrm{m}$. On further increase of field this biaxial state goes to a uniaxial dark state with the secondary directors in the neighboring

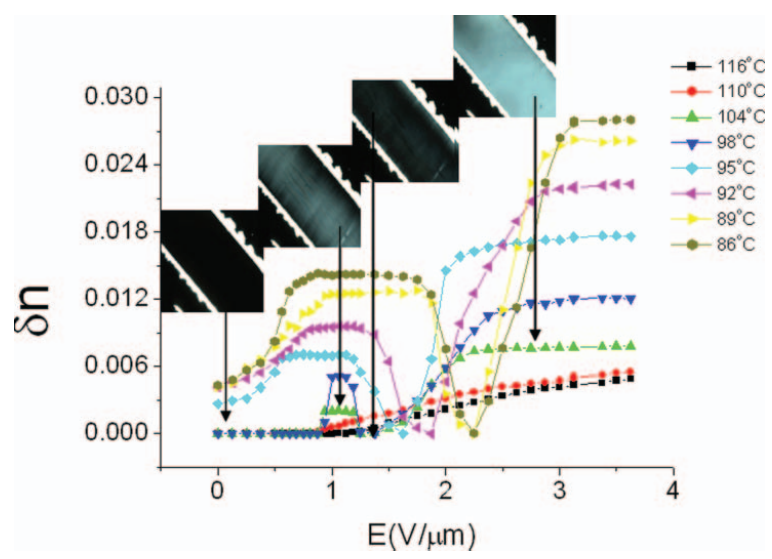

Figure 4. Electric field induced biaxiality measurements in PAL30 compound with decreasing temperature and the POM textures corresponding to the biaxiality value at $104^{\circ} \mathrm{C}$ (cell thickness $7 \mu \mathrm{m}$ and $\mathrm{f}=100 \mathrm{~Hz}$ ). 
smectic layers perpendicular to one another, similar to intermediate state in $\operatorname{SmAP}_{\mathrm{A}}$ and again goes to biaxial ferroelectric state and then saturates at higher field with $\delta n_{F}=0.007$. The $\mathrm{SmAP}_{\mathrm{AR}}$ phase under electric field exhibits two macroscopically uniaxial dark states separated by two biaxial bright states. All the materials under study (Table 1) show similar behavior in their $\mathrm{SmAP}_{\mathrm{A}}$ phase and the field response of $\mathrm{SmAP}_{\mathrm{R}}$ phases is similar for $\mathrm{CK} 64$ and PAL 30 samples.

\subsection{Spontaneous Polarization Measurements}

The polarization measurement is carried out in a $5.5 \mu \mathrm{m}$ planar cell for different applied square wave voltages $(2 \mathrm{~V}-58 \mathrm{~V})$ of $110 \mathrm{~Hz}$. The polarization is measured using the integral current technique. The important feature of this sample is the absence of $P$ in the entire temperature range when the field applied for its measurement is low.

The temperature dependence of macroscopic spontaneous polarization of PAL 30 compound is shown in Fig. 5. Application of $E$ above a threshold changes the distribution of the dipole moments (polar directors) and consequently gives rise to the net polarization $P$. In the higher temperature range of $\mathrm{SmAP}_{\mathrm{R}}$ phase, we find that the induced polarization is linearly proportional to the applied field. It is observed that $P(T)$ increases on approaching the $\mathrm{SmAP}_{\mathrm{R}}-\mathrm{SmAP}_{\mathrm{AR}}$ phase transition.

In the temperature range corresponding to $\mathrm{SmAP}_{\mathrm{AR}}$ and $\mathrm{SmAP}_{\mathrm{A}}$ phases, the net polarization is initially proportional to the applied field and then it jumps to a saturated value. It may be remarked that while the field-induced polarization shows a smooth-like normal dependence on $E$, the biaxiality (Figs. 3 and 4) shows sharp step-like behavior. This arises from the principal difference of the manner used in arriving at the results using the two techniques. The birefringence/biaxiality is measured for a localized small area in the cell, whereas the macroscopic polarization is measured over the entire area of the cell. The latter may consist of up to three different structures and hence the measured $P$ corresponds to an integrated response over the entire area.

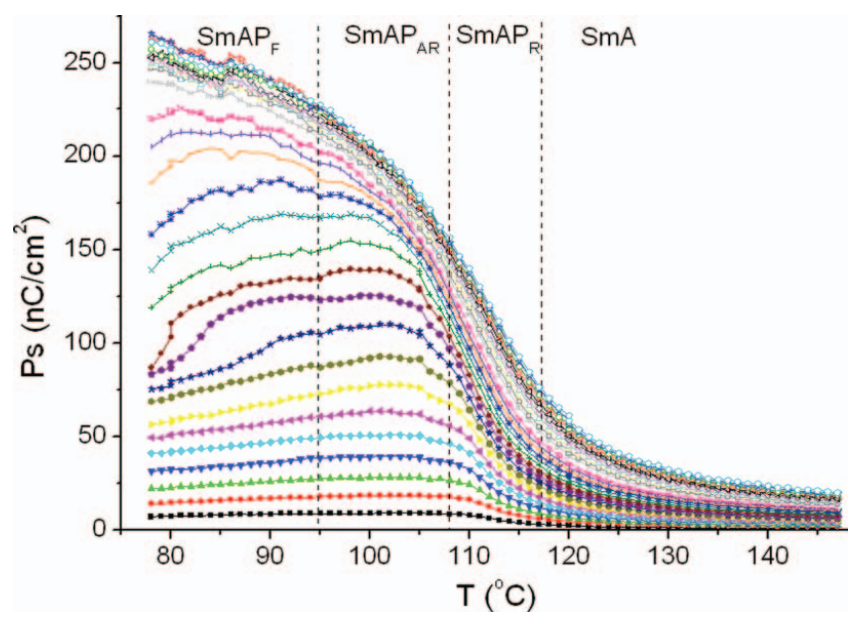

Figure 5. Temperature dependence of macroscopic spontaneous polarization in $5.5 \mu \mathrm{m}$ planar cell for different applied square wave voltages $(2 \mathrm{~V}-58 \mathrm{~V})$ of $100 \mathrm{~Hz}$ with a $2 \mathrm{~V}$ step. 

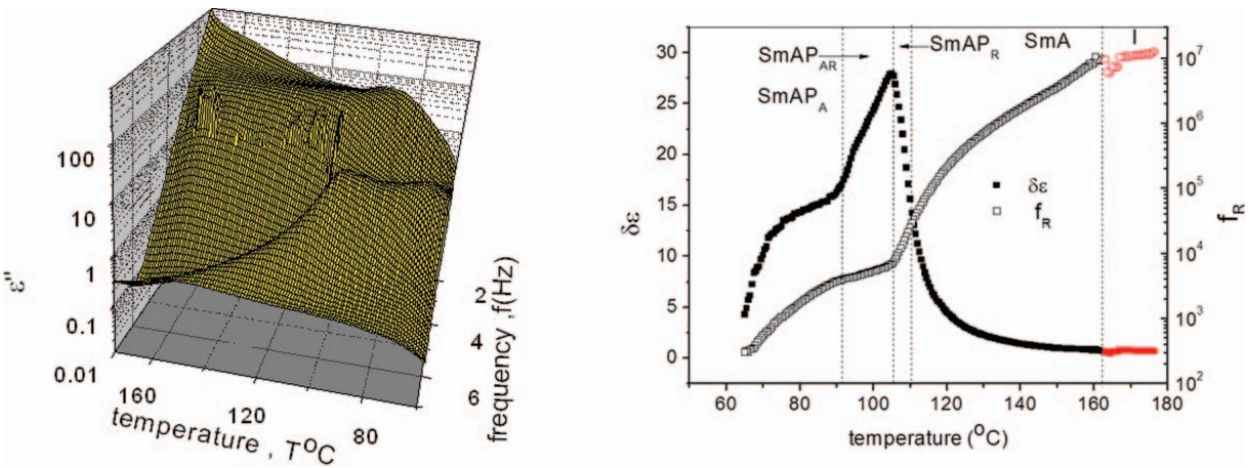

Figure 6. Dielectric behavior in $10 \mu \mathrm{m}$ planar cells (b) dielectric loss spectra $\left(\varepsilon^{\prime \prime}\right)$ and (b) dielectric amplitude $(\delta \varepsilon)$ and relaxation frequency $\left(\mathrm{f}_{\mathrm{R}}\right)$ of process $\mathrm{P} 2$ with temperature.

\subsection{Dielectric Spectroscopy}

The dielectric response of PAL-30 material over a frequency range of from $10 \mathrm{~Hz}$ to $10 \mathrm{MHz}$ is measured in $10 \mu \mathrm{m}$ (brass electrodes) planar cell using broadband high resolution Alpha-A frequency analyzer (Novocontrol GmbH, Germany). Figure 6(a) shows temperature dependence of dielectric loss spectra. On cooling from isotropic to $\mathrm{SmAP}_{\mathrm{A}}$ phase a relaxation peak is appeared in the lower frequency region, which is due to the contribution of longitudinal dipole moments. The dielectric spectrum is fitted to HavriliakNegami equation and the fitting parameters of the dominant process, e.g. dielectric strength (amplitude) and relaxation frequency are shown in the Fig. 6(b).

From this figure it is clearly seen that the dielectric amplitude $\Delta \varepsilon$ increases with lowering temperature and reaches a maximum at $\mathrm{SmAP}_{\mathrm{R}}-\mathrm{SmAP}_{\mathrm{AR}}$ transition.

\section{Conclusions and Perspectives}

In summary, field-induced biaxiality in different achiral orthogonal smectic phases in bent core banana-shaped liquid crystal compounds are investigated. The $\operatorname{SmAP}_{\mathrm{R}}$ phases is macroscopically uniaxial since secondary directors are randomly ordered in the absence of field, and the phase exhibits transformation from uniaxial to biaxial by an application of rather high electric field. In biaxial polar $\mathrm{SmAP}_{\mathrm{A}}$ phase molecules undergo biaxial-uniaxialbiaxial transformation with the strengthening electric fields, i.e., initial antiferroelectric structure transforms into ferroelectric state with increasing electric fields and it shows three optically distinguishable different levels. The $\mathrm{SmAP}_{\mathrm{AR}}$ phase shows an entirely different response compared to other smectic phases under study. The initial uniaxial state comes to biaxial state with field and with further increasing field strengths it shows again uniaxial dark state to biaxial bright state transformation. The dielectric measurements in $\mathrm{SmAP}_{\mathrm{AR}}$ phase is the evidence of antiferroelectric behavior of uniaxial state. The POM texture observation in CK64 and PAL 30 materials supports the corresponding biaxiality measurements. All materials under study (Table 1) show similar behavior in their $\mathrm{SmAP}_{\mathrm{A}}$ phase and the field response of $\mathrm{SmAP}_{\mathrm{R}}$ phases is similar for CK64 and PAL 30 samples.

\section{Acknowledgments}

The authors thank the SFI strand-1 project, EU Grant No. FP7-216025 BIND and SFI Grant No. RFP 06/RFP/ENE039 for funding this work. 


\section{References}

[1] Mayer, R. B., Liebert, L., Strzelecki, L., \& Keller, P. (1975). J. de Phys., 36, L69.

[2] Brand, H. R., Cladis, P. E., \& Pleiner, H. (1992). Macromolecules, 25, 7223.

[3] Niori, T., Sekine, T., Watanabe, J., Furukawa, T., \& Takezoe, H. (1996). J. Mater. Chem., 6, 1231.

[4] Eremin, A., Diele, S., Pelzl, G., Nadasi, H., Weissflog, W., Salfetnikova, J., \& Kresse, H. (2001). Phys. Rev. E., 64, 051707.

[5] Sadashiva, B. K., Reddy, R. A., Pratibha, R., \& Madhusudana, N. V. (2002). J. Mater. Chem., $12,943$.

[6] Schröder, M. W., Diele, S., Pancenko, N., Weissflog, W., \& Pelzl, G. (2002). J. Mater. Chem., $12,1331$.

[7] Murthy, H. N. S., \& Sadashiva, B. K. (2004). Liq. Cryst. 31, 567.

[8] Pociecha, D., Cepic, M., Gorecka, E., \& Mieczkowski, J. (2003). Phys. Rev. Lett., 91, 185501.

[9] Panarin, Y. P., Nagaraj M., Vij, J. K., Keith, C., \& Tschierske, C. (2010). Europhys. Lett., 92, 26002.

[10] Shimbo, Y., Takanishi, Y., Ishikawa, K., Gorecka, E., Pociecha, D., Mieczkowski, J., Gomola, K., \& Takezoe H. (2006). Jpn. J. Appl. Phys., 45, L282.

[11] Shimbo, Y., Gorecka, E., Pociecha, D., Araoka, F., Goto, M., Takanishi, Y., Ishikawa, K., Meieczkowski, J., Gomola, K., \& Takezoe, H. (2006). Phys. Rev. Lett., 97, 113901.

[12] Gomola, K., Guo, L., Pociecha D., Araoka, F., Ishikawa, K., \& Takezoe, H. (2010). J. Mater. Chem., 20, 7944-7952. 